\title{
LE TÉMOIGNAGE DES MONNAIES sur l'orfèvrerie chypriote classique
}

\section{Evangéline MARKOU}

\begin{abstract}
On the Cypriot coins of the Classical period three types of earrings (disk, pendant and circular earring) and two types of necklaces (pearl necklace and torque) can be observed. The purpose of this paper is to present the typology of the above through the testimony of coins and to compare these representations to other works of art, such as jewellery, sculpture, terracottas, vase-painting and coinages minted in other parts of the Greek world, in order to discuss the influences and the general tendencies of the period.
\end{abstract}

Déchiffrer les détails des images sur la surface réduite des monnaies est souvent une tâche difficile. En raison des détériorations subies au moment de la frappe, lors de leur circulation et, surtout, pendant l'enfouissement, celles-ci sont souvent mal conservées, et même si l'on arrive à repérer les types représentés, les détails qui les composent ne sont pas toujours clairs. Néanmoins, les exemplaires bien conservés qui ont survécu et le rapprochement entre les types monétaires et des représentations sur des supports différents permettent d'acquérir plus d'informations sur les expressions d'un art local et sur les modes en vigueur à l'époque étudiée.

Les têtes et les bustes portant des bijoux qui font l'objet de cette contribution datent majoritairement $\mathrm{du} \mathrm{IV}^{\mathrm{e}}$ siècle av. J.-C. Les émissions frappées au cours du v viècle représentent en effet, dans leur grande majorité, des animaux, réels ${ }^{1}$ ou fictifs ${ }^{2}$, des

1. Par exemple le bouc couché à Salamine (BMC Cyprus, p. 46, nº 1 sq., pl. IX, fig. 1 sq. ; Traité II.1, col. 575, no 919, pl. XXVI, fig. 1 sq. ; Traité II.2, col. 697, n 1129, pl. CXXVII, fig. 1 et suivantes ; Zapiti, Michaelidou 2007, p. 36-38, pl. I, fig. 1-6, 9-15, 18); le taureau et l'aigle à Paphos (BMC Cyprus, p. 36, no 4, pl. VII, fig. 4 sq. ; Traité II.2, col. 781, no 1278, pl. CXXXIII, fig. 18 sq. ; Kraay 1976, p. 377, pl. 63, nos 1088-1090 ; Zapiti, Michaelidou 2007, p. 67, no 1, pl. IV, fig. 1 sq.) ; le lion à Amathonte (BMC Cyprus, p. 2, no 6, pl. I, fig. 6 sq. ; Traité II.2, col. 769, $\mathrm{n}^{\circ} 1254$, pl. CXXXII, fig. 11 sq. ; Zapiti, Michaelidou 2007, p. 77, $\mathrm{n}^{\circ} 1$, pl. V, fig. 1 sq.) ; le poulpe au revers d'un atelier chypriote indéterminé (A. Destrooper-Georgiades, «Le trésor de Larnaca (IGCH 1272) réexaminé », RDAC 1984, p. 157, pl. XXX, fig. 102-104).

2. Voir le sphinx à Idalion (BMC Cyprus, p. 24, no 1, pl. V, fig. 1 sq. ; Traité II.2, col. 761, pl. CXXXII, fig. 1 sq. ; Kraay 1976, p. 378, pl. 64, nºs 1096-1098 ; Zapiti, Michaelidou 2007, p. 99 , $\mathrm{n}^{\circ} 2$, pl. VII, fig. 2 et sq. 
scènes animalières ${ }^{3}$, divers symboles ${ }^{4}$ ou des scènes mythologiques ${ }^{5}$. En revanche, les

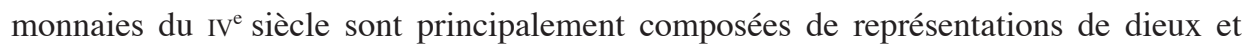
de déesses, suivant une tendance générale que l'on observe dans la plupart des ateliers monétaires de l'époque classique ${ }^{6}$.

Les parures visibles sur les monnaies chypriotes sont des colliers et des boucles d'oreilles, dont on peut distinguer des types différents, deux pour les colliers - les colliers de perles et le torque -, trois pour les boucles d'oreille - les anneaux, les pendentifs simples ou élaborés et les boucles en forme de disque.

\section{Boucles d'oreilles}

\section{Boucle en forme de disque}

La boucle d'oreille en forme de disque est la version la plus simple de ce bijou. On la voit sur les émissions des rois de Lapéthos qui datent du $\mathrm{v}^{\mathrm{e}}$ siècle, en particulier sur le sicle d'un roi incertain qui représente, au droit, la tête d'Aphrodite diadémée à droite, l'œil de face, avec une boucle en forme de disque, au revers celle d'Athéna coiffée d'un casque corinthien dans un carré creux ${ }^{7}$ (Fig. 1).

Un autre exemple, fourni par le sicle du roi Sidqémilk, a été émis dans ce même royaume au milieu du $\mathrm{v}^{\mathrm{e}}$ siècle. Il représente au droit la tête d'Athéna coiffée du casque corinthien, avec un collier de perles et une boucle en forme de disque ; au revers est figurée la tête de la même déesse vue de face, coiffée du casque attique orné de deux oreilles de taureau et de deux hautes crêtes, avec un collier ${ }^{8}$. Enfin, on connaît dans ce royaume une autre émission, datée des années 425 , qui représente au droit la tête

3. Ainsi le lion dévorant un cerf au revers des émissions des rois de Kition (BMC Cyprus, p. 10 sq. , $\mathrm{n}^{\text {os }}$ 10-71, et 75-82, pl. III, fig. 1-14 et pl. IV, fig. 1-17 et 20-24 ; Traité II.2, col. 739, $\mathrm{n}^{\circ}$ 1204, pl. CXXX, fig. 19 sq. ; Zapiti, Michaelidou 2007, p. 56, nº 5, pl. V, fig. 5 sq.).

4. Comme l'ankh au revers des émissions salaminiennes ( BMC Cyprus, pl. IX, p. 48-52, nos $12-$ 32, fig. 10-19 et pl. X, fig. 1-10 ; Traité II.1, col. 579, no $933^{\text {bis }}$, pl. XXVI, fig. 10 sq. ; Traité II.2, col. 697, n 1129, pl. CXXVII, fig. 1 sq. ; Zapiti, Michaelidou 2007, p. 37, nº 9, pl. I, fig. 9 sq.

5. Voir les émissions des rois de Marion, Sasmas et Stasioikos I, qui représentent au revers de leur monnayage en argent Phrixos et le bélier (Sasmas) et Europe sur le taureau (Stasioikos II). Pour l'identification des types iconographiques voir L. Lacroix, «Deux traversées miraculeuses sur les monnaies de Marion en Chypre », dans Études d'archéologie numismatique, Paris, 1974, p. 53-65. Pour un exemplaire des monnaies de Sasmas voir Zapiti, Michaelidou 2007, p. 85, pl. VI, fig. 1-2 ; pour des monnaies de Stasioikos I voir W. Schwabacher, « The coins of the Vouni treasure reconsidered », NR Cy XII, 1981, p. 36-37, $\mathrm{n}^{\text {os }}$ 5-21.

6. O. Picard, «Images des dieux sur les monnaies grecques », MEFRA 103, 1991, p. 223-233.

7. Traité II.2, col. 821-822, n 1358, pl. CXXXVI, fig. 10 ; BMC Cyprus, p. 29, n 3, pl. VI, fig. 3 ; Kraay 1976, p. 378, pl. 63, nº 1092.

8. Traité II.2, col. 821-824, nos 1361-1363, pl. CXXXVI, fig. 12-14 ; BMC Cyprus, p. 30-31, $\mathrm{n}^{\text {os }}$ 7-9, pl. VI, fig. 6-8 ; Dembski 1999, p. 213 et 217, nº 107. 
d'Athéna coiffée du casque corinthien, avec une boucle d'oreille du même type, au revers la tête d'Héraclès coiffée de la peau de lion ${ }^{9}$.

Au IV ${ }^{\mathrm{e}}$ siècle une boucle en forme de disque est visible sur la tête d'Athéna représentée au revers des émissions en or du roi Nikoklès de Salamine ${ }^{10}$.

Il est intéressant de remarquer que, dans la plupart de ces cas, c'est Athéna qui porte ce type de bijou, que l'on retrouve représenté sur la tête d'Athéna en calcaire du Musée de Nicosie, datée du V $V^{e}$ siècle, qui a été découverte dans le temple d'Athéna au palais de Vouni ${ }^{11}$. Ce même type de boucle d'oreille est figuré sur d'autres sculptures de la même époque, par exemple sur des têtes coiffées d'un sakkos ${ }^{12}$ (Fig. 2), même si souvent les têtes chypriotes portent des boucles en forme de rosette ${ }^{13}$. On pourrait se demander si le bijou représenté sur les émissions chypriotes n'est pas une rosette simplifiée, mais le témoignage des monnaies ne permet pas de discerner les détails qui indiqueraient que les graveurs des coins monétaires ont voulu y reproduire une boucle plus élaborée en forme de rosette plutôt qu'une boucle en forme de disque simple.

Pour les boucles d'oreille en forme de disque, les parallèles ne manquent pas dans l'orfèvrerie antique ${ }^{14}$. Des bijoux de ce type ont été découverts entre autres à Chypre ${ }^{15}$, à Rhodes ${ }^{16}$, à Kymè ${ }^{17}$, en Égypte ${ }^{18}$ et en Étrurie ${ }^{19}$.

9. C.M. Kraay, «The Celenderis Hoard », NC 1962, p. 11, pl. II, nº 16 ; Zapiti, Michaelidou 2007, p. 94, $\mathrm{n}^{\circ} 3$, pl. VII, fig. 3 . Voir aussi la monnaie mise en vente chez Classical Numismatic Group, vente 72,14 juin $2006, \mathrm{n}^{\circ} 852$.

10. Traité II.2, col. 713-714, $\mathrm{n}^{\text {os }}$ 1160-1162, pl. CXXVIII, fig. 1-3; BMC Cyprus, p. 58-59, n $^{\text {os }}$ 61-63, pl. XI, fig. 22-24 ; Coll. Giabra Pierides, p. 319, nº 425 ; Zapiti, Michaelidou 2007, p. $49, \mathrm{n}^{\mathrm{os}} 16-17$, pl. II, fig. $16-17$.

11. SCE III, p. 103, pl. XL, fig. 1-3 ; V. Karageorghis, Greek Gods and Heroes in Ancient Cyprus, Athènes, 1998, p. 109, fig. 66.

12. Hermary 1989, p. 352 sq., surtout les $\mathrm{n}^{\text {os }} 701,702,710,712,714-716,719,723-724,729-730$, 732 ; Coll. Giabra Pierides, p. 159, $\mathrm{n}^{\text {os }} 200-201$ et p. 162-163, $\mathrm{n}^{\text {os }} 206-207$.

13. Hermary 1989 , p. $356, n^{\circ} 713$; p. 358 , fig. 719 ; p. 359 , fig. 721 ; p. 360 , fig. $723-724$; p. 362 , fig. 728 .

14. Sur ce sujet voir l'analyse détaillée de Van den Driessche 1971, p. 73-96.

15. Pierides 1971, p. 30-31, pl. 20, fig. 1-4 ; Van den Driessche 1971, p. 86-87, fig. 5 et p. 90 ; Deppert-Lippitz 1985, p. 185-187, fig. 133.

16. Van den Driessche 1971, p. 90-92 ; Williams, Ogden 1994, p. 98, nº 52.

17. Deppert-Lippitz 1985, p. 187, fig. 134.

18. Van den Driessche 1971, p. 95.

19. Voir les deux disques en or et en cristal découverts dans une tombe du ve siècle en Étrurie dans G.M.A. Richter, «Four Notable Acquisitions of the Metropolitan Museum of Art », AJA 40, 1940, p. 434-439, fig. 7-9. Voir aussi la paire conservée au Field Museum of Natural History à Chicago : I.M. Richard, «Etruria », dans T. Hackens, R. Winkes (éds.), Gold Jewelry. Craft, Style and Meaning from Mycenae to Constantinopolis. Catalogue of the exhibition held at the Museum of Art, Rhode Island School of Design from February 24, 1983-April 3, 1983, Louvain, 1983, p. 101, n. 25. Pour les disques découverts en Étrurie voir également Marshall 1911, p. 136-140, 
La présence de ce bijou sur les monnaies chypriotes renvoie certainement aussi aux représentations d'Athéna sur les monnaies d'Athènes, un des monnayages grecs les plus riches et les plus célèbres, qui fut largement diffusé au cours du $\mathrm{v}^{\mathrm{e}}$ siècle ${ }^{20}$, où la déesse porte une boucle en forme de disque tout au long des $\mathrm{v}^{\mathrm{e}}-\mathrm{IV}^{\mathrm{e}}$ siècles ${ }^{21}$ (Fig. 3) ; ce bijou est à repérer également sur les émissions des cités de l'Ionie ${ }^{22}$, dont Phocée, et surtout sur celles de Cnide depuis le vi ${ }^{\mathrm{e}}$ siècle ${ }^{23}$.

\section{Boucles à pendentifs}

Le deuxième type attesté sur les monnaies chypriotes est celui des pendentifs, que l'on trouve sous deux variantes : le pendentif simple et le pendentif plus élaboré, composé de trois éléments suspendus.

\section{Pendentif simple}

Sur les didrachmes et les hémidrachmes d'étalon dit « rhodien » d'Évagoras II de Salamine, Aphrodite, représentée au droit coiffée d'une couronne tourelée, et Athéna au revers, coiffée d'un casque corinthien, portent toutes deux à l'oreille des pendentifs similaires ${ }^{24}$ (Fig. 4). Les bijoux, qu'on retrouve aussi sur les fractions en or du même roi ${ }^{25}$, sont formés d'un disque attaché au lobe, d'où tombe un pendentif conique en forme de pyramide renversée.

Toujours à Salamine, au revers des didrachmes en argent de Pnytagoras, Artémis, identifiable par le carquois qu'elle porte sur l'épaule, les cheveux relevés et noués en chignon, porte ce même type de bijou, dont le pendentif s'achève par une perle ${ }^{26}$.

$\mathrm{n}^{\text {os }}$ 1414-1426, pl. XX ; R.A. Higgins, Greek and Roman jewellery, 2éd., Berkeley, 1980, p. 140, pl. 32 A-B.

20. Sept tétradrachmes athéniens ont été découverts à Chypre, dans le trésor de Dali (date d'enfouissement vers 425-400) : M. Thompson, O. Mørkholm, C.M. Kraay, An Inventory of Greek Coin Hoards, New York, 1973, $\mathrm{n}^{\circ} 1275$ et C. Flament, Le monnayage en argent d'Athènes, de l'époque archaïque à l'époque hellénistique (c. 550-c. 40 av. J.-C.), Louvain, 2007, p. 184.

21. Pour un aperçu de ce monnayage voir Flament, op. cit., particulièrement p. 112-117 le tableau des styles de droit du $v^{\mathrm{e}}$ siècle.

22. Voir un groupe de monnaies, émis probablement en Ionie du nord, dans H.A. Cahn, «Ionische Damen », dans R. Ashton, S. Hurter, G. Le Rider, R. Bland (éds.), Studies in Greek Numismatics in memory of Martin Jessop Price, Londres, 1998, p. 59-63, et surtout pl. 16, fig. 1-14 (agrandissements).

23. H.A. Cahn, Knidos, Die Münzen des sechsten und des fünften Jahrhunderts v. Chr., Berlin, 1970, p. 105 et pl. 12, fig. 1 et suivantes (agrandissements).

24. Traité II.2, col. 717-718, $\mathrm{n}^{\text {os }}$ 1169-1170, pl. CXXVIII, fig. 9-10 ; Coll. Giabra Pierides, p. 320-321, no 427 ; Dembski 1999, p. 218 et p. 222, n 111.

25. Voir l'agrandissement d'un $1 / 10^{\mathrm{e}}$ de statère de ce roi dans Zapiti, Michaelidou 2007, p. 50, $\mathrm{n}^{\circ} 18$, pl. II, fig. 18 .

26. Traité II.2, col. 725-726, nº 1186, pl. CXXVIII, fig. 28. 
L'émission en or du roi de Paphos Timarchos nous propose une variante de cette forme de pendentif. Au droit est représentée la tête d'Aphrodite, au revers une colombe debout à droite, avec une légende en syllabaire chypriote qui se lit « du roi Timarchos » (pa si / ti ma ra ko), avec en exergue les lettres grecques П A, l'ethnique de la ville ${ }^{27}$. Au droit, Aphrodite, coiffée d'une haute stéphanè décorée de fleurs, porte un pendentif formé de quatre perles en forme de croix. Un parallèle proche se trouve sur les tétradrachmes en argent émis par Cnide dans la première décennie du IV ${ }^{\mathrm{e}}$ siècle ${ }^{28}$.

Ce type de bijou est courant dans l'orfèvrerie grecque depuis la fin du viI ${ }^{\mathrm{e}}$ siècle ${ }^{29}$ et devient très populaire au cours $\mathrm{du} \mathrm{VI}^{\mathrm{e}}$ siècle, comme en témoignent de nombreuses images, dont celle de la coupe à figures rouges d'Oltos à Tarquinia, qui date des années $515-510^{30}$. Son usage continue également aux $\mathrm{V}^{\mathrm{e}}$ et $\mathrm{IV}^{\mathrm{e}}$ siècles, période pendant laquelle la décoration du bijou est plus élaborée ; il est figuré sur un grand nombre de sculptures chypriotes $\mathrm{du} \mathrm{IV}^{\mathrm{e}}$ siècle, dont des têtes en terre cuite coiffées de hauts kalathoi, conservées à Copenhague ${ }^{31}$, et sur la tête de la collection De Clercq au Louvre ${ }^{32}$ (Fig. 5).

Ce bijou, sans doute de grand prix, rappelle une boucle qui combine une rosette au niveau du lobe et une pyramide renversée - le lien entre les deux parties étant formé par un oiseau -, dont la provenance est soit l'île de Chypre, soit un atelier indéterminé qui se situe à l'est ${ }^{33}$ (Fig. 6). Il me paraît fort probable, même si la comparaison entre le bijou réel et sa représentation sur les monnaies et les sculptures parait peu pertinente, que, pour ces compositions, l'inspiration provient bien de ce type de bijou.

Le pendentif d'oreille simple n'est pas une exclusivité chypriote. On le retrouve sur le monnayage d'autres ateliers du monde grec, comme sur les émissions de Lampsaque

27. S.W. Grose, Catalogue of the McClean Collection of Greek coins, vol. 3, Cambridge, 1929, p. $309, \mathrm{n}^{\circ} 9158$, pl. 331, fig. 16 .

28. R.H.J. Ashton, P. Kinns, K. Konuk, A.R. Meadows, « The Hecatomnus Hoard ( $\mathrm{CH}$ 5.17, 8.96, 9.387) », dans A. Meadows, U. Wartenberg (éds.), Coin Hoards volume IX. Greek Hoards, Royal Numismatic Society, Special Publication $\mathrm{n}^{\circ}$ 35, Londres, 2002, p. 117, $\mathrm{n}^{\circ} 14$ sq., pl. 12, fig. 14a sq. Pour le type, voir aussi B.V. Head, Catalogue of the Greek coins of Caria, Cos, Rhodes, $\& c$., Bologne, 1964, p. 87, nº 24, pl. XIV, fig. 6.

29. Benaki 1999, p. 161, n 48, fig. 109 ; Despini 1996, p. 97, fig. 69-70 ; Deppert-Lippitz 1985, p. 125-126, fig. 74-75.

30. Benaki 1999, p. 160, nº 108 ; Deppert-Lippitz 1985, p. 125, fig. 71.

31. V. Karageorghis et al., Ancient Cypriote Art in Copenhagen. The collections of the National Museum of Denmark and the NY Carlsberg Glyptotek, Nicosie, 2001, p. 71-73, $\mathrm{n}^{\mathrm{os}} 130-133$; S. Fourrier, A. Queyrel, L'art des modeleurs d'argile. Antiquités de Chypre. Coroplastique, vol. 2, Paris, 1998 , p. 531, n $^{\circ} 869$; p. 532, n$^{\circ} 871$; p. 536, n $^{\circ} 884$; p. 573 , no 888.

32. Hermary 1989 , p. $409, n^{\circ} 832$.

33. Williams, Ogden 1994, p. 241, no 176 ; Marshall 1911, p. 181, nº 1666-7, pl. XXX. 
en Mysie ${ }^{34}$ (Fig. 7) ou celles de Nagidos en Cilicie ${ }^{35}$, datées de la première moitié du $\mathrm{IV}^{\mathrm{e}}$ siècle.

\section{Pendentifformé de trois éléments suspendus}

Parmi les pendants d'oreille attestés sur les monnaies chypriotes, c'est la version plus élaborée, constituée de trois éléments suspendus, qu'on trouve le plus souvent.

À Kition, on voit ce type de pendentif au droit des émissions en bronze attribuées au roi Milkyaton, qui représentent la tête d'Aphrodite ${ }^{36}$. La déesse apparaît pour la première et unique fois sur les émissions de ce royaume, dont l'iconographie est par ailleurs très stable depuis le tout début du monnayage. Aphrodite est coiffée d'une haute stéphanè décorée ; les cheveux retombent en chignon sur la nuque, elle porte un collier et un pendentif composé de trois éléments suspendus. Au revers, on retrouve l'iconographie habituelle des rois de Kition : Héraklès debout à droite, tenant l'arc de la main gauche et, de la droite, brandissant la massue au-dessus de sa tête. Dans le champ à gauche, on lit la lettre phénicienne « $M »$. Toutefois, le bronze étant un métal qui résiste mal au temps, il nous est pratiquement impossible de discerner les détails de ce bijou. Il en va parfois de même pour les émissions en argent.

Sur une monnaie de Marion du IV siècle, émise par le roi Stasioikos II, on distingue une autre variante de ce type de pendentif. Au droit est représentée la tête de Zeus lauré, au revers celle d'Aphrodite coiffée d'une couronne de myrte, portant un collier de perles et un pendentif d'oreille formé d'un disque d'où tombent trois pendentifs dont les extrémités semblent être décorées de petits globules ${ }^{37}$. Il est intéressant de remarquer que, sur cette monnaie, la partie supérieure du bijou, au niveau du lobe, se rapproche beaucoup des représentations de boucles d'oreilles en forme de disque ${ }^{38}$ (Fig. 8).

Ce type de boucle est également associé aux représentations d'Aphrodite sur les émissions de Paphos, où la déesse est représentée parée de la forme élaborée de ce bijou à triple pendentif, notamment sur un sicle de Timarchos qui représente au droit la tête d'Aphrodite coiffée d'une stéphanè plate, ornée de palmettes, avec collier et pendentif à l'oreille, au revers une colombe à droite ${ }^{39}$.

À Salamine, on retrouve des pendentifs de ce type sur les didrachmes en argent dits « rhodiens » de Pnytagoras, qui représentent au droit la tête d'Aphrodite, au revers celle

34. Traité II.2, col. 1375, $\mathrm{n}^{\circ}$ 2554-2556, pl. CLXXI, fig. 21-24; W. Wroth, Catalogue of the Greek Coins of Mysia in the British Museum, Londres, 1892, p. 81, n 29, pl. XIX, fig. 7 ; Coll. Gulbenkian II, p. 682-683, pl. LXVI, fig. 682-683.

35. Coll. Gulbenkian II, p. 98, nº 802, pl. LXXXVI, fig. 802.

36. Traité II.2, col. 755-758, n 1230, pl. CXXXI, fig. 22-23; Classical Numismatic Group, vente Triton V, 15 janvier 2002, n 527.

37. Dembski 1999, p. 218-219, nº 108 ; BMC Cyprus, p. 1xi (h) et pl. XX, 13.

38. Voir supra, p. 412.

39. Traité II.2, col. 799-800, no 1319, pl. CXXXV, fig. 4 ; BMC Cyprus, p. 44, n 47, pl. VIII, fig. 9. 
d'Artémis, comme on l'a vu plus haut ${ }^{40}$. Sur certaines variantes, Artémis porte clairement à l'oreille un pendentif formé de trois éléments suspendus, comme Aphrodite au droit ${ }^{41}$. De même, sur les statères en or du même roi, Aphrodite, coiffée d'une couronne tourelée au droit, porte ce même type de bijou ${ }^{42}$; au revers est figuré un buste masculin sur lequel je reviendrai plus loin ${ }^{43}$.

Les statères en or de Nikokréon ${ }^{44}$ ainsi que les fractions en or de Ménélas ${ }^{45}$, toujours à Salamine, perpétuent la même iconographie, avec de légères variations dans la zone du pendentif. Sur les monnaies en or, et surtout sur les monnaies lourdes, notamment les statères, on arrive à voir clairement les détails des bijoux, même sur les exemplaires issus de coins usés, car l'or se conserve mieux dans la durée.

Il est intéressant de prendre en considération, comme parallèle de ces représentations de bijoux sur les monnaies, la tête d'Aphrodite conservée à Gratz, qui date du IV siècle ${ }^{46}$. La déesse est coiffée d'une couronne tourelée et porte à l'oreille un triple pendentif très proche de l'iconographie monétaire salaminienne. Sur les émissions de Nagidos en Cilicie, qui datent des années 380-360, on retrouve un motif semblable : c'est, cette fois, Tyché qui est représentée avec les mêmes attributs, la couronne tourelée et la variante de la boucle d'oreille à triple pendentif ${ }^{47}$.

Cette même forme de pendentif se voit mieux sur une émission en or du roi de Paphos Nikoklès, qui représente au droit la tête d'Aphrodite de face, au revers un aigle debout à gauche, les ailes repliées ${ }^{48}$ (Fig. 9). Cette représentation de la déesse de face permet de

40. Voir supra, p. 414 et n. 26.

41. BMC Cyprus, p. 63, nos 77-78, pl. XII, fig. 12-13; Dembski 1999, p. 223-224, nº 112 ; Zapiti, Michaelidou 2007, p. 50, n 23, pl. II, fig. 23.

42. BMC Cyprus, p. 62, no 76, pl. XII, fig. 11 ; Zapiti, Michaelidou 2007, p. 50, n 22, pl. II, fig. 22.

43. Voir infra, p. 419.

44. Traité II.2, col. 727-728, n 1188 , pl. CXXIX, fig. 10 ; BMC Cyprus, p. cxiii, nº 1, pl. XXIV, 21.

45. Traité II.2, col. 729-730, pl. CXXIX, fig. 14 ; BMC, Cyprus, p. cxiii-cxiv, pl. XXIV, 23.

46. C. Beer, «A head of a marble Tyche from Idalion », CCEC 32, 2002 (Hommage à Marguerite Yon), p. 369-385 ; G. Erath-Koiner, « Aphrodite in Graz. Eine Mauerkroneträgerin aus Zypern im Landesmuseum Joanneum », Schild van Steier 18, 2005, p. 27-35, surtout p. 32-33 ; ead., "On the way to Hellenism: Late Classical and early Hellenistic portraits from Cyprus », dans P. Flourentzos (éd.), Proceedings of the International Archaeological Conference: From Evagoras I to the Ptolemies. The Transition from the Classical to the Hellenistic Period in Cyprus, Nicosia 29-30 November 2002, Nicosie, 2007, p. 201-205, fig. 1-2.

47. Göktürk 2000, p. 143, pl. XVII, fig. 5. Voir également la monnaie mise en vente chez Peus, vente 376,29 octobre $2003, n^{\circ} 494$.

48. Traité II.2, col. 801-802, n 1325, pl. CXXIX, fig. 16 ; BMC Cyprus, p. lxxvii, pl. XXII, fig. 7. 
rendre le type du bijou avec une précision beaucoup plus grande ; on voit plus clairement ici la partie en forme de disque fixée au lobe, la partie centrale en forme de pyramide renversée et les deux chaînes extérieures plus fines, dont les extrémités sont décorées de petits globules. Les pendentifs comme ceux de la collection Cesnola, mis au jour à Kourion, qui datent d'environ 350-300 ${ }^{49}$ (Fig. 10), constituent sans doute les modèles qui ont inspiré les graveurs de ce coin monétaire.

Ce type de boucle d'oreille a été découvert non seulement en Grèce de l'Est, comme la célèbre paire de Kymè ${ }^{50}$, mais aussi dans d'autres régions du monde grec ${ }^{51}$.

Les boucles à triple pendentif sont très courantes dans l'iconographie monétaire de l'époque classique. Les premières appartiennent au monnayage de Syracuse, où elles apparaissent, avec des variantes intéressantes, sur plusieurs émissions représentant la tête de la nymphe Aréthuse ${ }^{52}$ (Fig. 11), qui a inspiré plusieurs ateliers du monde grec ${ }^{53}$. On les voit également sur les sicles d'Amisos du Pont dans les années $350{ }^{54}$, sur des oboles en argent anépigraphes du $\mathrm{IV}^{\mathrm{e}}$ siècle en Cilicie ${ }^{55}$ et sur les statères d'or de Lampsaque, qui datent de la première moitié du $\mathrm{IV}^{\mathrm{e}}$ siècle ${ }^{56}$.

49. Williams, Ogden 1994, p. 242-243, n 177 ; V. Karageorghis et al., Ancient Art from Cyprus. The Cesnola Collection in the Metropolitan Museum of Art, New York, 2000, n 394.

50. Williams, Ogden 1994, p. 96-97, nos 49-50 (Kymè).

51. Des parallèles proches ont été découverts, entre autres, à Madytos (Williams, Ogden 1994, p. 110-111, fig. 63), dans le tumulus de Bliznitza (ibid., p. 181, fig. 116), à Syracuse (DeppertLippitz 1985, p. 164-165, fig. 115), en Italie du Sud à Tarente (Despini 1996, p. 116-117 et p. 235, fig. 95-96 ; Deppert-Lippitz 1985, p. 187-188, fig. 135) et dans l'île de Kalymnos (Despini 1996, p. 98 et p. 226-227, fig. 71). Voir également un parallèle proche conservé au Musée Bénaki, à Athènes, de provenance inconnue, qui date de la fin du Ive siècle : Benaki 1999, p. 188-189, nº 60, fig. 132 ; Despini 1996, p. 100-101 et p. 227, fig. 73-74.

52. Pour le monnayage de Syracuse du $\mathrm{v}^{\mathrm{e}}$ siècle voir la monographie de E. Boehringer, Die Münzen von Syrakus, Berlin-Leipzig, 1929. Pour les boucles concernées ici, voir les décadrachmes des années 400-390 dans Sylloge Nummorum Graecorum, The collection of the American Numismatic Society, Part 5, Sicily III : Syracuse-Siceliotes, New York, 1988, ${ }^{\circ \text { s }} 361-375$; aussi, un tétradrachme des années 410-400 dans G.K. Jenkins, Monnaies grecques, Fribourg 1972, p. 188, $\mathrm{n}^{\circ} 417$.

53. Voir par exemple les similitudes de la tête d'Aréthuse sur les monnaies de Syracuse à la tête au droit des émissions de Locris (Locri Opuntii) dans la Péloponnèse dans Coll. Gulbenkian II, p. 37-38, nº 489 , pl. LI, fig. 489 et suivantes.

54. SNG Cop, Bosporus-Bithynia, $\mathrm{n}^{\circ \mathrm{s}}$ 120-128 ; Traité II.2, col. 1527, $\mathrm{n}^{\circ}$ 2953, pl. CLXXXV, fig. 11. Voir également la monnaie mise en vente chez Baldwin et Münzen \& Medaillen, The New York Sale III, 7/XII/2000, nº 143.

55. Voir la monnaie mise en vente chez Classical Numismatic Group, vente 73, 13 septembre $2006, \mathrm{n}^{\circ} 413$.

56. A. Baldwin, «The Gold coinage of Lampsacus », Journal International d'Archéologie Numismatique 5, 1902, p. 24, nº 36, pl. III, fig. 23 ; Coll. Gulbenkian II, p. 75, nº 688, pl. LXVI, fig. 688 . 


\section{Anneaux d'oreille}

Enfin, l'anneau n'est présent que sur le buste masculin au revers des émissions d'or des derniers rois de Salamine à partir de Pnytagoras (Fig. 12), chez son successeur Nikokréon et jusqu'à Ménélas ${ }^{57}$.

Cet anneau d'oreille est décoré de plusieurs globules sur son pourtour et dans la partie centrale. Le parallèle le plus proche dans l'orfèvrerie antique, qui date du $\mathrm{IV}^{\mathrm{e}}$ siècle, provient d'Achalgori, en Géorgie ${ }^{58}$ (Fig. 13). Le style oriental de ce bijou se rapproche des boucles achéménides provenant de Lydie, de Syrie, de Suse ou de Babylone ${ }^{59}$, mais aussi de boucles de Kourion ${ }^{60}$, datées après le milieu du IV ${ }^{\mathrm{e}}$ siècle, dont des parallèles en argent ont été découverts en Syrie du Nord ${ }^{61}$.

Il n'existe pas de parallèle proche de ce type de boucle d'oreille dans la numismatique des époques archaïque et classique. Néanmoins parmi les émissions des ateliers monétaires orientaux, on repère des anneaux d'oreilles sur les émissions de Datames (378-372) à Tarse $^{62}$ et de Mazaios (361/360-334) à Myriandros de Cilicie ${ }^{63}$.

\section{Colliers}

\section{Collier de perles}

Le collier composé d'une rangée de perles est le genre de collier que l'on retrouve le plus souvent, on peut même dire presque exclusivement, sur les monnaies chypriotes. On le voit associé à des têtes d'Aphrodite, d'Athéna ou d'Artémis sur des émissions

57. Il apparaît pour la première fois sur les oboles en argent émis par Évagoras II (Traité II, col. 159-172 ; BMC, Cyprus, p. cix-cx, pl. XXIV, 18-19). Pour le commentaire historique et l'analyse de la continuité iconographique sur les monnayages en or de derniers rois de Salamine voir Markou 2006, p. 139-143. Pour les boucles d'oreilles sur les têtes masculins voir A. Hermary, E. Markou, «Les boucles d'oreilles bijoux masculins à Chypre et en Méditerranée orientale (VII $\mathrm{e}_{-}$ IV ${ }^{\mathrm{e}}$ siècles avant J.-C.) », CCEC 33, 2003, p. 211-236.

58. E. Rehm, Der Schmuck der Achämeniden, Münster, 1992, p. 146, fig. 114, F.79 ; Markou 2006, p. 137, fig. 4.

59. B. Musche, Vorderasiatischer Schmuck von den Anfängen bis zur Zeit der Achaemeniden (ca. 10000-330 v. Chr.), Leyde, 1993, type 6, p. 270-274, pl. CIV ; Rehm, op. cit., p. 145-147, fig. 114, F. 79.

60. Williams, Ogden 1994, p. 230, no 162 et p. 240, nº 175 . Voir aussi une boucle découverte à Chypre dans SCE IV.2, p. 162-163, fig. 34, nº3.

61. Williams, Ogden 1994, p. 240. Pour les parallèles syriens, voir K.R. Maxwell-Hyslop, Western Asiatic Jewellery c. 3000-612 B.C., Londres, 1971, p. 268, pl. 217 et 256.

62. SNG France 2, Cilicie, pl. 10, n ${ }^{\text {os } 278-281 ; ~ G o ̈ k t u ̈ r k ~ 2000, ~ p . ~ 145, ~ n o ~} 25$ (Tarkumuwa), pl. XIX, fig. 5.

63. SNG France 2, Cilicie, pl. 16, n 429 ; E.T. Newell, « Myriandros-Alexandreia Kat'Isson », AJN 53-II, 1919, p. 16, nº 4, fig. 20 ; Göktürk 2000, p. 146-147, nº 35, pl. XX, fig. 5. 
de plusieurs rois dans différents royaumes ${ }^{64}$. Mais, sur la fraction en argent du roi de Paphos dont le nom termine en (...)damos ${ }^{65}$, Aphrodite représentée au droit, coiffée d'une couronne très ornée, porte autour du cou un double collier de perles ou un collier à pendentifs, tandis qu'au revers figure une colombe battant des ailes ${ }^{66}$ (Fig. 14).

Cette représentation, rare sur les monnaies chypriotes, est en accord avec les images de la déesse dans la sculpture locale, comme par exemple la tête en calcaire d'Idalion datant du début du $\mathrm{v}^{\mathrm{e}}$ siècle ${ }^{67}$, qui porte autour du cou un collier composé de plusieurs rangées de perles avec un fermoir central rectangulaire. Il peut s'agir de la partie supérieure d'une parure très ornementée, comme celle de la statue en calcaire de Trikomo, qui date du $\mathrm{VI}^{\mathrm{e}}$ siècle (Fig. 15); elle est composée, en plus de rangs en «tour de cou », de colliers longs, formés de perles rondes ou biconiques, avec un pendentif central ${ }^{68}$, dont un parallèle proche est fourni par un collier chypriote découvert à Arsos, aujourd'hui conservé au Musée de Nicosie, qui date de la fin du viI ${ }^{\mathrm{e}}$ siècle ${ }^{69}$.

Un pendentif de ce genre est peut-être figuré sur les tiers et les douzièmes de statère d'or du roi Nikoklès de Salamine, qui représentent au droit le buste d'Aphrodite et au revers la tête d'Athéna (Fig. 16) ${ }^{70}$. Aphrodite est coiffée d'un haut calathos décoré de personnages aux bras ouverts, dont les bandelettes retombent sur les côtés. Si l'on compare le buste d'Aphrodite sur la monnaie à la sculpture du Musée du Louvre ${ }^{71}$, datée $\mathrm{du} \mathrm{v}^{\mathrm{e}}$ siècle, on verra clairement que les deux têtes portent le même type de couronne, et les bandelettes, visibles de profil sur la statue, sont reproduites de la même manière sur le coin monétaire ${ }^{72}$ (Fig. 17).

64. Presque toutes les représentations des têtes des déesses déjà mentionnées portent un collier de perles autour du cou, dont Athéna (à Salamine par Nikoklès, voir supra p. 413 et n. 10) Artémis et Aphrodite (à Salamine par Pnytagoras, voir supra p. 417 et n. 41), Aphrodite (à Kition par Milkyaton, voir supra p. 416 et n. 36 ; à Marion par Stasioikos II, voir supra p. 416 et n. 37).

65. E. Babelon dans Traité II.2, col. 797-798, n 1316, pl. IX, fig. 6, propose de lire sur cette monnaie le nom Échetimos. G.F. Hill dans BMC, Cyprus, p. lxxvi, propose de lire Charidamos. D'après O. Masson, ICS, p. $122, \S 28$, la lecture Échetimos n'est pas possible; en revanche, la proposition de G.F. Hill est plus probable.

66. BMC, Cyprus, p. 43, n 46, pl. VIII, fig. 8 ; Traité II.2, col. 797-798, n 1316, pl. CXXXV, fig. 2.

67. Karageorghis 2005, p. 189, nº 242 ; Hermary 1989, p. 330, nº 650.

68. Karageorghis 2005, p. 221, no 326 ; Hermary 1989, p. 400, no 811.

69. Pierides 1971, p. 27-28, pl. XV, fig. 1 ; Benaki 1999, p. 94, fig. 53.

70. BMC, Cyprus, p. 58-59, $\mathrm{n}^{\text {os }}$ 61-63 et pl. XI, 22-24 ; Traité II.2, $\mathrm{n}^{\text {os }} 1160-1162$ et pl. CXXVIII, 1-3 ; Zapiti, Michaelidou 2007, p. 49, $\mathrm{n}^{\text {os }} 16-17$, pl. II, fig. 16-17.

71. Hermary 1989 , p. $402, n^{\circ} 815$.

72. E. Markou, «L'originalité chypriote à travers l'iconographie des monnaies d'or du IV $\mathrm{V}^{\mathrm{e}}$ siècle avant J.-C. », dans P. Flourentzos (éd.), Proceedings of the International Archaeological Conference: From Evagoras I to the Ptolemies. The Transition from the Classical to the Hellenistic Period in Cyprus, Nicosia 29-30 November 2002, Nicosie, 2007, p. 288. 
La comparaison entre les deux objets permet une remarque additionnelle. Il est fort probable que la partie obscure qui se trouve dans le champ situé au-dessous du menton de la déesse sur les émissions de Nikoklès signale l'extension du collier de perles, dont une partie est visible au dessus de l'épaule d'Aphrodite. Sur certains coins, il paraît y avoir dans cette partie de la monnaie deux rangées de globules, qui représentent sans doute des perles, sur d'autres semble figurer un élément allongé qui pourrait représenter la partie pendante du collier. Mais la vue de profil rend la tentative de représentation d'un objet aussi complexe peu réussie, ce qui explique pourquoi on ne retrouve pas sur les monnaies la variété des colliers produits par les ateliers d'orfèvrerie locaux qui sont abondamment représentés sur le statuaire locale ${ }^{73}$. En revanche, après le milieu du IV siècle, au moment où la présentation de la tête de face ou de trois-quarts devient à la mode dans l'iconographie monétaire ${ }^{74}$, différentes variétés de colliers apparaissent sur les monnaies grecques. L'explication de ce phénomène est aussi, je pense, d'ordre technique, puisqu'il est plus facile pour les graveurs de coins de représenter les détails complexes d'un collier de face que de profil, comme en témoignent les monnaies ciliciennes du IV siècle ${ }^{75}$. Les émissions en électrum de Carthage démontrent néanmoins qu' au cours du III $^{\mathrm{e}}$ siècle (290270) des artistes arriveront à un haut niveau de création artistique (Fig. 18) ${ }^{76}$.

\section{Torque}

Le torque est très rarement représenté sur les monnaies qui datent de l'époque classique. Le seul exemple à Chypre de la représentation de ce bijou, purement oriental, est fourni par les émissions en or des derniers rois de Salamine ${ }^{77}$. Sur les monnaies, le collier est représenté ouvert dans sa partie antérieure ; ses extrémités semblent décorées d'avant-trains d'animaux, comme le torque de Suse qui est contemporain ${ }^{78}$ (Fig. 19).

73. Hermary 1989 , p. 322 , n ${ }^{\text {os }} 634-635 ;$ p. 337 , nos $669-670 ;$ p. $340, n^{\circ} 676 ;$ p. $342, n^{\circ} 680$; p. $351, \mathrm{n}^{\mathrm{o}} 698$; p. $366, \mathrm{n}^{\text {os }} 739-740 ;$ p. $403, \mathrm{n}^{\circ} 818 ;$ p. $407, \mathrm{n}^{\circ} 828$.

74. K.P. Erhart, The Development of the Facing Head Motif on Greek Coins and its Relations to Classical Art, Thèse de Doctorat, Harvard University, 1979.

75. SNG France 2, Cilicie, pl. 8-9, ${ }^{\text {os }}$ 241-250 (Pharnabaze) ; pl. 9-10, $\mathrm{n}^{\text {os }}$ 258-277 (Datame) ; Coll. Gulbenkian II, p. 98, nº 804, pl. LXXXVI, fig. 804.

76. Pour les émissions de Carthage voir G.K. Jenkins, R.B. Lewis, Carthaginian Gold and Electrum Coins, Royal Numismatic Society Special Publication n ${ }^{\circ}$ 2, Londres 1963, p. 101-104, groupe VI (c. 290-280), nos 307-340, pl. 13-14 ; Gulbenkian I, p. 124, nº 380, pl. XLII, fig. 380.

77. Voir supra p. 419 et n. 57. Analyse détaillée dans Markou 2006, p. 137-139, fig. 5-6.

78. A. Caubet et al., La cité royale de Suse. Trésors du Proche-Orient ancien au Louvre, Paris, 1994, p. 245, no 171. 


\section{Conclusion}

Les rois de Chypre, seuls responsables de leurs émissions monétaires, semblent suivre, au cours du $\mathrm{IV}^{\mathrm{e}}$ siècle, les tendances de l'époque : ils représentent des têtes et des bustes parés de bijoux et ils empruntent, adoptent, amalgament des éléments de l'orfèvrerie, de la glyptique et de l'iconographie des autres monnayages.

Les éléments purement orientaux qu'on peut reconnaître clairement sur leurs émissions se limitent à la présence de l'anneau d'oreille et du torque, attestés à Salamine. Au cours du $\mathrm{IV}^{\mathrm{e}}$ siècle, la mise en commun des formes et la multiplicité des influences rendent plus difficile de distinguer la provenance précise de telle ou telle forme de bijou, surtout dans la mesure où les détails de fabrication ou de décoration ne sont pas toujours apparents sur les monnaies.

De même, il est pratiquement impossible de savoir si l'inspiration des graveurs des coins monétaires vient directement de l'orfèvrerie ou passe par l'intermédiaire de la sculpture ou des représentations des mêmes bijoux sur d'autres monnayages voisins, ou célèbres.

Le collier de perles est le type de collier que l'on retrouve le plus souvent sur les monnaies chypriotes, comme c'est le cas dans la plupart des émissions monétaires de l'époque classique du monde grec. Cela s'explique en partie par la difficulté de représenter de profil les colliers composés de plusieurs rangées et de pendentifs, éléments qu'on s'attendrait à trouver au moins sur les représentations d'Aphrodite à Chypre, d'après les témoignages de la sculpture locale.

Les boucles d'oreille sont, au contraire, représentées de façons plus variées. Si le disque, souvent lié aux représentations d'Athéna, est un bijou qui apparaît presque exclusivement sur les émissions du $v^{\mathrm{e}}$ siècle, le pendentif semble devenir le bijou préféré de l'iconographie monétaire $\mathrm{du} \mathrm{IV}^{\mathrm{e}}$ siècle. Sa version élaborée, avec trois éléments suspendus, qui est attestée sur un nombre important de monnaies classiques émises dans divers ateliers du monde grec, caractérise la majorité des monnayages chypriotes postérieurs au milieu du IV ${ }^{\mathrm{e}}$ siècle.

Musée Numismatique, Athènes

\section{ABREVIATIONS}

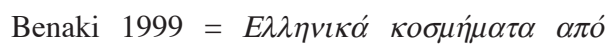

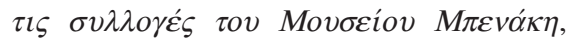
Athènes.

BMC Cyprus = G.F. Hill, Catalogue of the Greek coins of Cyprus in the British Museum, Londres, 1904.

Coll. Giabra Pierides = E. Zapiti, L. Michaelidou, «The Coins », dans V. Karageorghis et al., Ancient Art from Cyprus in the Collection of George and Nefeli Giabra Pierides, Athènes, 2002, p. 316-327.
Coll. Gulbenkian I = E.S.G. Robinson, $A$ Catalogue of the Calouste Gulbenkian Collection of Greek Coins. Part I. Italy, Sicily, Carthage, Lisbonne, 1971.

Coll. Gulbenkian II = G.K. Jenkins, A Catalogue of the Calouste Gulbenkian Collection of Greek Coins. Part II. Greece to East, Lisbonne, 1989.

ICS = O. Masson, Les inscriptions chypriotes syllabiques, 2e édition, Paris, 1983. 
SCE III = E. Gjerstad et al., The Swedish Cyprus expedition: Finds and results of the Excavations in Cyprus 1927-1931, vol. III, Stockholm, 1937.

SCE IV.2 = E. Gjerstad, The Swedish Cyprus expedition. Vol. 4: part 2: the CyproGeometric, Cypro-Archaic and CyproClassical periods, Stockholm, 1948.

SNG ANS 5, III = Sylloge Nummorum Graecorum, The collection of the American Numismatic Society, Part 5, Sicily III : Syracuse-Siceliotes, New York, 1988.

SNG Cop, Bosporus-Bithynia $=$ Sylloge Nummorum Graecorum. The Royal Collection of Coins and Medals. Danish National
Museum, Bosphorus-Bithynia, Copenhague, 1944.

SNG France 2, Cilicie $=$ Sylloge Nummorum Graecorum France 2, Cabinet des Médailles. Cilicie, Paris, 1993.

Traité II.1 = E. Babelon, Traité des monnaies grecques et romaines, deuxième partie, description historique, tome premier : Les monnaies grecques depuis les origines jusqu'aux guerres médiques, Paris, 1907.

Traité II. 2 = E. Babelon, Traité des monnaies grecques et romaines, deuxième partie, description historique, tome deuxième, Paris, 1910.

\section{BIBLIOGRAPHIE}

Dembski (G.), 1999, «Griechische Münzen aus Zypern in Wien », dans A. BernhardWalcher, G. Dembski, K. Gschwantler, V. Karageorghis, Die Sammlung zyprischer Antiken im Kunsthistorischen Museum, Wien, Vienne.

DePPERT-LiPPITZ (B.), 1985, Griechischer Goldschmuck, Mayence.

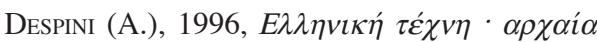

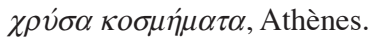

GöKTÜRK (T.), 2000, « Small coins from Cilicia and Surroundings ", dans O. Casabonne (éd.), Mécanismes et Innovations monétaires dans l'Anatolie achéménide. Numismatique et histoire. Actes de la Table Ronde Internationale d'Istanbul, 22-23 mai 1997, Varia Anatolica XII, Paris, p. 143-151.

Hermary (A.), 1989, Musée du Louvre, Département des antiquités orientales. Catalogue des antiquités de Chypre: Sculptures, Paris.

Karageorghis (J.), 2005, Kypris. The Aphrodite of Cyprus. Ancient Sources and Archaeological Evidence, Nicosie.

KraAY (C.M.), 1976, Archaic and Classical Greek Coins, Londres.
Markou (E.), 2006, «Problèmes iconographiques du monnayage d'or des derniers rois de Salamine au Ive siècle avant Jésus-Christ ", dans S. Fourrier, G. Grivaud (éds.), Identités croisées en un milieu méditerranéen : le cas de Chypre (Antiquité - Moyen Âge), Rouen, p. 135-151.

Marshall (F.H.), 1911, Catalogue of the Jewellery, Greek, Etruscan, and Roman : in the Departments of Antiquities, British Museum, Londres.

Pierides (A.), 1971, Jewellery in the Cyprus Museum, Nicosie.

VAN DEN DRIESSChe (B.), 1971, «Une forme grecque de boucles d'oreilles portées par les Korai de l'Acropole », Revue des Archéologues et Historiens d'art de Louvain 4, p. 73-96.

Williams (D.), Ogden (J.), 1994, Greek Gold. Jewelry of the Classical World, New York.

ZAPITI (E.), MichaELIDOU (L.), 2007,

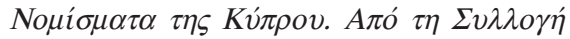

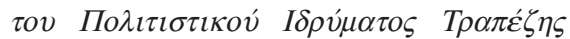
Kv́ $\pi \rho \circ v$, Nicosie. 


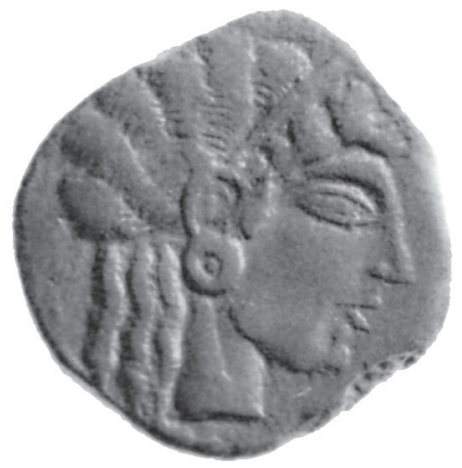

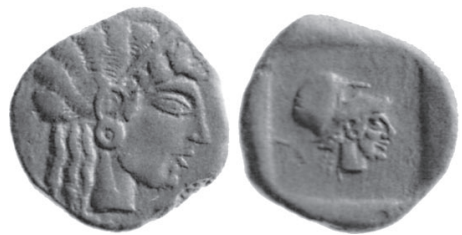

Figure 1. D'après Kraay 1976, p. 378, pl. $63, n^{\circ} 1092$.

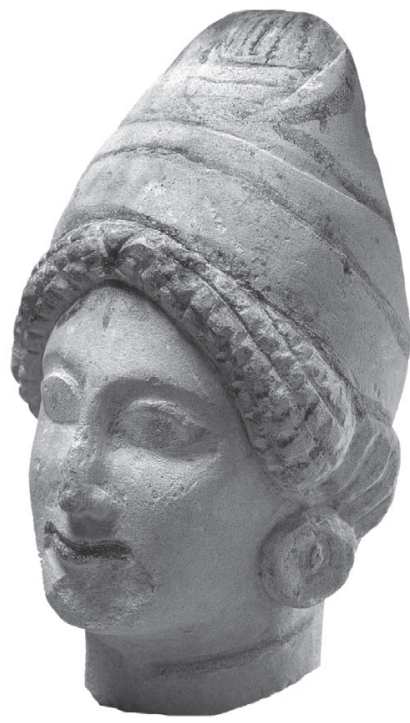

Figure 2. D'après Coll. Giabra Pierides, p. $163, \mathrm{n}^{\circ} 207$.

Figure 3. Tétradrachme $d u v^{e}$ siècle.

D'après Coll. Gulbenkian II, p. 43, pl. LIII, n 521.
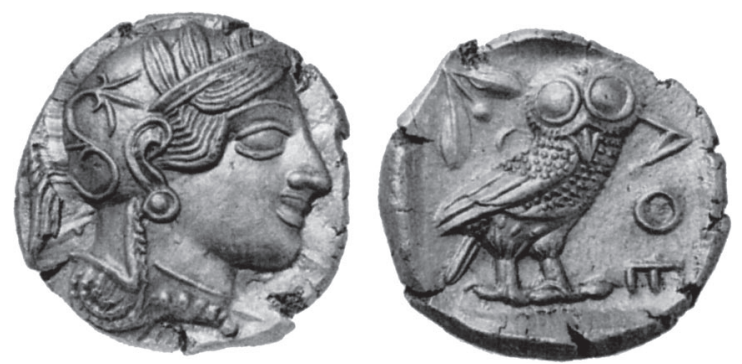

Figure 4. D'après Dembski 1999, p. 218 et $222, n^{\circ} 111$.
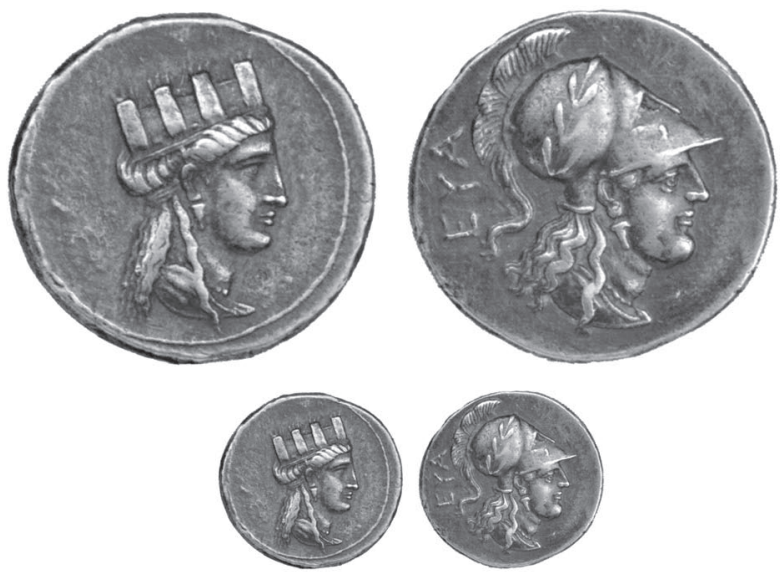

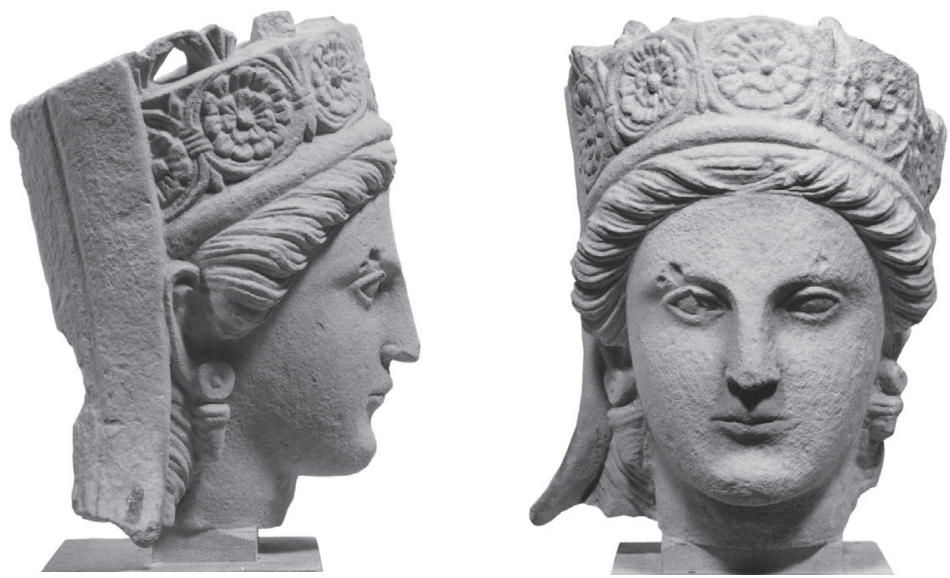

Figure 5. D'après Hermary 1989, p. 409, n 832.

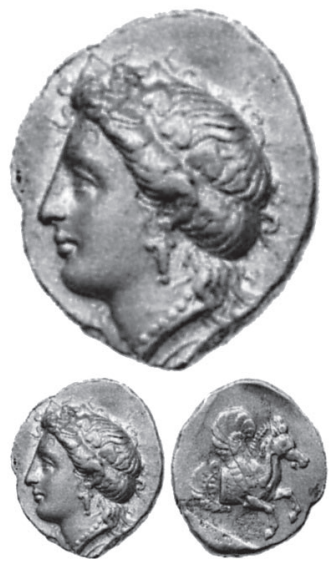

Figure 7. Statère d'or du IV siècle. D’après Coll. Gulbenkian II, p. 74, pl. LXVI, n 683.
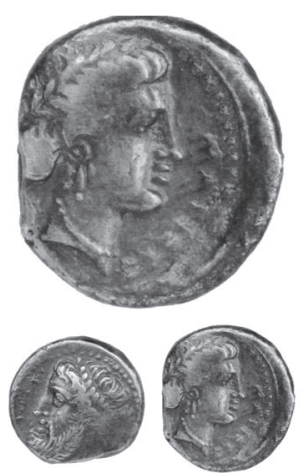

Figure 8. D'après Dembski 1999 , p. $218-219$, n$^{\circ} 108$.

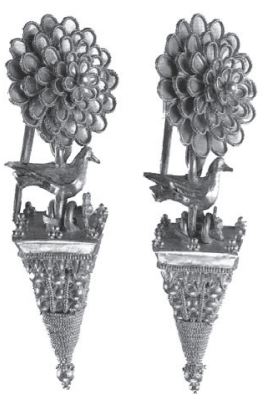

Figure 6. D'après Williams, Ogden 1994 , p. $241, \mathrm{n}^{\circ} 176$.

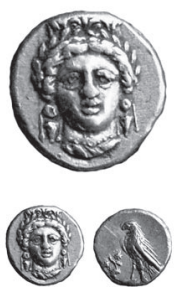

Figure 9. D'après Traité II.2, col. 801-802, n 1325 , pl. CXXIX, fig. 16.

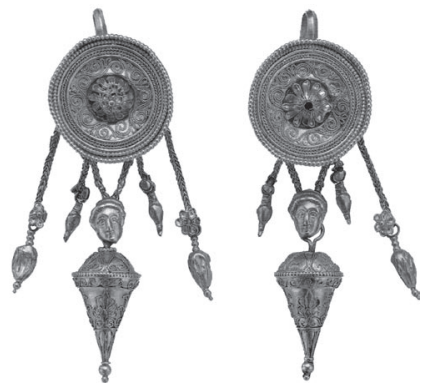

Figure 10. D'après Williams, Ogden 1994, p. 242-243, n 177. 

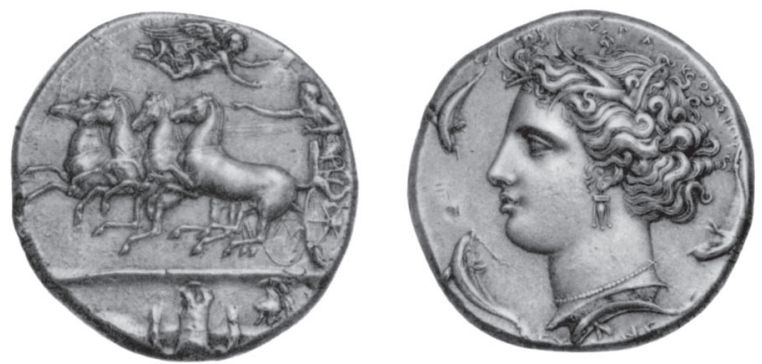

Figure 11. Décadrachme des années 410-375.

D'après Coll. Gulbenkian I, p. 105, pl. XXXVI, nº 310.
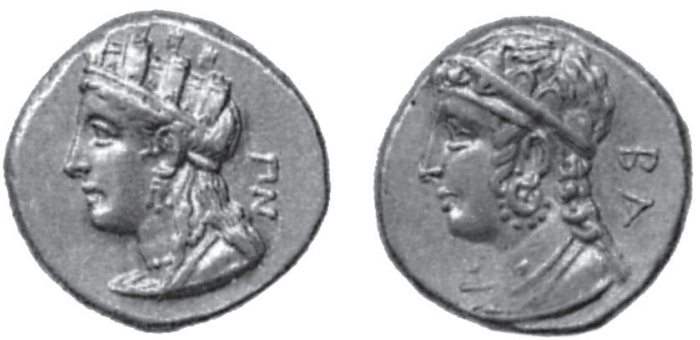

Figure 12. Statère d'or. D'après Coll. Gulbenkian II, p. 101, pl. LXXXVI, fig. 813.
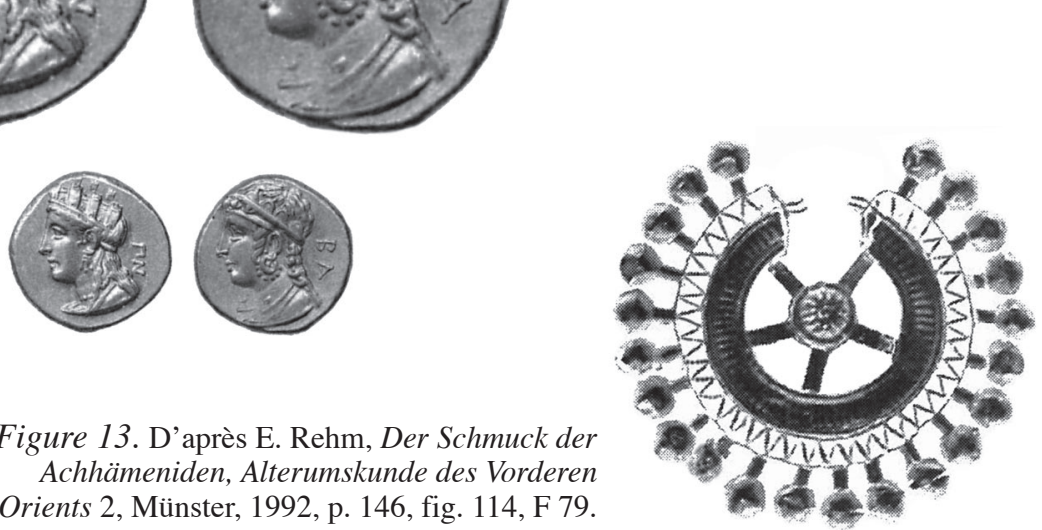

Figure 13. D'après E. Rehm, Der Schmuck der Achhämeniden, Alterumskunde des Vorderen Orients 2, Münster, 1992, p. 146, fig. 114, F 79.
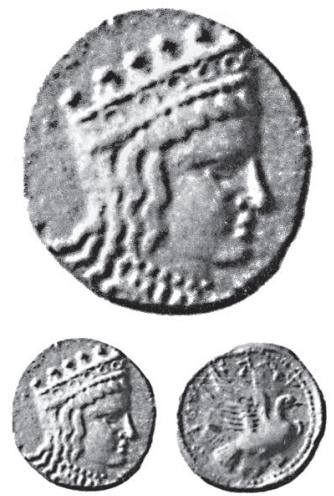

Figure 14. D'après Traité II.2, col. 797798, n 1316, pl. CXXXV, fig. 2.

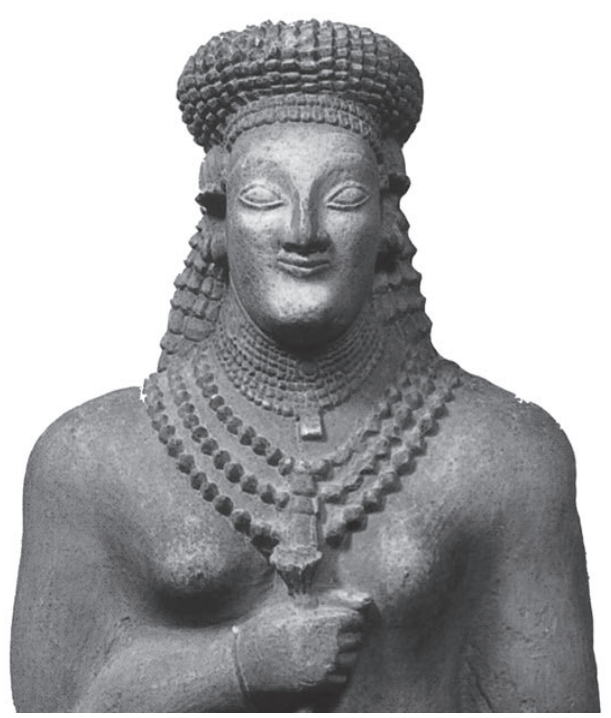

Figure 15. D'après Karageorghis 2005, p. 221, nº 326. 


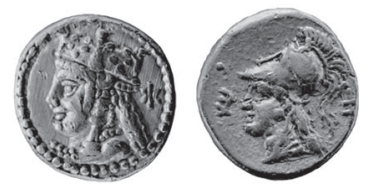

Figure 16. 1/3 de statère d'or.

D'après Zapiti, Michaelidou 2007, p. 49, n 16, pl. II, fig. 16.
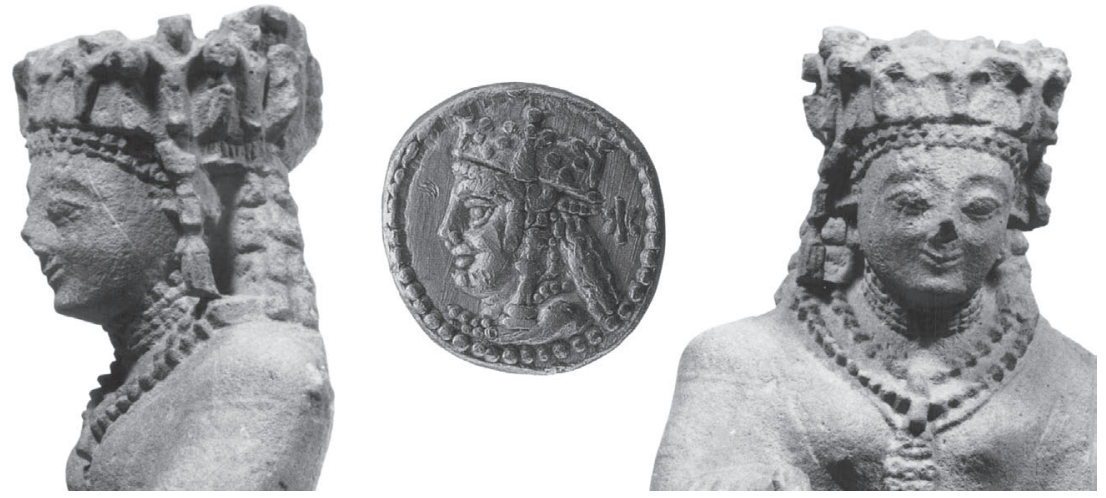

Figure 17. La sculpture.

D'après Hermary 1989, p. 402, n 815 ; agrandissement du droit de la figure 16.

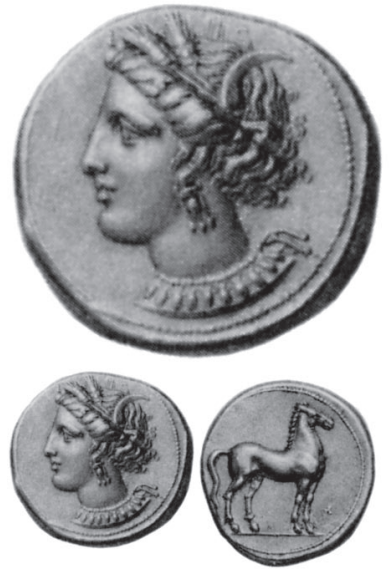

Figure 18. D'après Coll. Gulbenkian I, p. $124, \mathrm{n}^{\circ} 380$, pl. XLII, fig. 380.

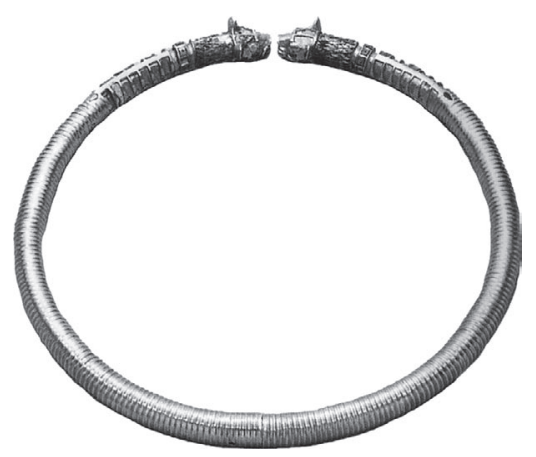

Figure 19. D'après A. Caubet et alii, La cité royale de Suse. Trésors du Proche Orient ancien au Louvre, Paris, 1994, p. 245, nº 171. 